\title{
Numerical Analysis of Cavitating Flow of Magnetic Fluid in a Vertical Venturi Channel*
}

\author{
Jun ISHIMOTO** and Shinichi KAMIYAMA**
}

\begin{abstract}
The fundamental multiphase flow characteristics of the two-dimensional cavitating flow of magnetic fluid in a vertical venturi channel under a strong nonuniform magnetic field are numerically predicted to realize the further development and high performance of the new type of a two-phase fluid driving system using magnetic fluids. First, the governing equations of the cavitating flow of a hexane-based magnetic fluids based on the unsteady thermal nonequilibrium two-fluid model are presented and several two-phase flow characteristics are numerically calculated taking into account the effect of the strong nonuniform magnetic field. Based on the numerical results, the two-dimensional structure of the cavitating flow and cloud cavity formation of the magnetic fluid through a vertical venturi channel are shown in detail. The numerical results demonstrate that effective two-phase magnetic driving force and fluid acceleration at the venturi channel are obtained by the practical use of the magnetization of the working fluid. Also clarified is the precise control of the cavitating flow of magnetic fluid that is possible by effective use of the magnetic body force which acts on cavitation bubbles.
\end{abstract}

Key Words: Magnetic Fluid, Multiphase Flow, Cavitation, MHD, Internal Flow, Bubble

\section{Introduction}

The precise investigation of cavitation or two-phase flow phenomena of magnetic fluid is very interesting and important not only as the basic studies on hydrodynamics of magnetic fluids, but also for finding solutions to problems related to the development of practical engineering applications of magnetic fluids, such as new energy conversion system using two-phase flows of magnetic fluid which was proposed by one of the authors ${ }^{(1),(2)}$.

The principle of such a fluid driving system is that the flow is accelerated in the region of the converging venturi nozzle section, and the cavitation inception is induced at the downstream point of the throat of a diverging venturi nozzle section due to a pressure decrease. Furthermore, the flow is additionally accelerated not only by the pumping effect of the cavitation bubbles, but also by the rise of magnetic pressure induced by the unbalance of magnetic body forces in the single- and two-phase flow regions under a nonuniform magnetic field. The idea of using a two-phase flow system originated from the twophase liquid-metal MHD power generation system which

* Received 7th December, 2004 (No. 04-4241)

** Institute of Fluid Science, Tohoku University, Transdisciplinary Fluid Integration Research Center, 2-1-1 Katahira, Aoba-ku, Sendai 980-8577, Japan was proposed and developed by Petrick and Branover ${ }^{(3)}$. After their proposal, we reported the results of a theoretical study which demonstrated the possibility of using an electrically conducting magnetic fluid ${ }^{(4),(5)}$ as a working fluid in a liquid-metal MHD power generation system ${ }^{(6)}$, where it was shown that a better driving force or pressure rise than that of the conventional system was obtained by using an electrically conducting magnetic fluid as the working fluid due to the practical application of the magnetization of the fluid. According to these previous studies, it is likely that high performance of the power generation system is possible by applying the electrically conducting magnetic fluid to a working fluid in the two-phase LMMHD $^{(7),(8)}$ power generation system.

In the past few years, theoretical and experimental studies on the basic characteristics of two-phase flow of magnetic fluid were conducted and the possibility of flow control or effective driving force generation by magnetic force in the new energy conversion system using twophase flow was confirmed ${ }^{(1),(2)}$. It was also concluded that stabilization of two-phase flow is possible by effective application of the magnetic force of the fluid ${ }^{(2),(9)}$. In the application of the two-phase flow of the magnetic fluid to an actual fluid transport apparatus, it is important to determine a simple and effective method to generate the twophase flow state for the improvement of the total performance of a fluid driving system using multiphase flow. 
However, conventional two-phase flow systems essentially require a powerful heat source or gas-injection equipment for generation of the boiling two-phase flow or gas-liquid two-phase flow state. Additionally, the research on methods for the production of the two-phase magnetic fluid flow state have not been precisely focused, and only a few studies have so far been made on the basic mechanism of the cavitating flow due to the difficulty of confirming experimental and theoretical results in high-speed two-phase magnetic fluid flow with phase change. In order to overcome these difficulties, we contrived a new type of two-phase fluid driving and acceleration system by using cavitating flow of magnetic fluid, which has conspicuous feature that there are no-heat sources or no-additional gasinjection devices except for venturi channel. Based on an advanced mathematical model, which takes the effect of two-phase magnetic body force acting on cavitating magnetic fluid flow state into consideration, we herein develop a new method for analyzing cavitating flow.

In the present study, two-dimensional characteristics of cavitating flow of magnetic fluid in a vertical venturi channel with phase change are numerically investigated to demonstrate the further development and high performance of the two-phase fluid acceleration system or fluid transport applications. First, the governing equations of cavitating flow of magnetic fluid based on the unsteady two-fluid model in the generalized curvilinear coordinates system are presented, and then several cavitating flow characteristics are numerically calculated, taking into account the effect of the strong nonuniform magnetic field.

\section{Nomenclature}

$a^{(\mathrm{i})}$ : interfacial area concentration per unit volume

$C_{D}:$ drag coefficient

$C_{V}$ : virtual mass coefficient

$c_{p}:$ specific heat at constant pressure

$c_{0}$ : sound velocity

$D$ : inlet width of duct

$e:$ specific internal energy

$e^{i j k}:$ permutation symbol

$g_{r}^{i}$ : contravariant vector of gravitational acceleration

$g^{i j}$ : fundamental metric tensor

$H$ : strength of magnetic field

$H^{i}$ : contravariant vector of magnetic field

$H_{\max }:$ maximum strength of magnetic field

$h:$ specific enthalpy

$k$ : heat transfer rate

$k_{B}$ : Boltzmann's constant

$M:$ strength of magnetization

$M^{i}$ : contravariant vector of magnetization

$N$ : number density

$p:$ absolute pressure

$q$ : heat flux

$q^{i}:$ contravariant heat flux vector
$R$ : radius

$\mathfrak{R}$ : gas constant

$T:$ absolute temperature

$T_{s}$ : saturation temperature

$t$ : time

$u^{i}, u^{j}, u^{k}:$ contravariant velocity

$u_{i} u_{j}, u_{k}$ : covariant velocity

Greek symbols

$\alpha$ : volume fraction

$\beta$ : dynamic viscosity

$\Gamma$ : phase generation density

$\gamma:$ surface tension

$\eta$ : transverse coordinate

$\kappa:$ ratio of specific heat

$\lambda:$ thermal conductivity

$\mu_{0}$ : magnetic permeability in vacuum

$v$ : kinematic viscosity

$\xi$ : longitudinal coordinate

$\rho:$ density

$\Omega^{i}$ : contravariant angular velocity vector

$\omega^{i}$ : contravariant vorticity vector

$\nabla_{j}$ : covariant differential

Subscripts and superscripts

()$_{c}$ : condensation

()$_{e}$ : evaporation

( $)_{(\mathrm{ex})}$ : exit section of the duct

()$_{g}:$ gas phase

()$^{i},()^{j},()^{k}$ : contravariant component

()$_{i},()_{j},()_{k}$ : covariant component

()$^{(i)}:$ interface

( ) (in) : inlet section of the duct

()$_{l}$ : liquid phase

()$_{s}:$ saturation

()$_{T}$ : two-phase

( $)^{\xi}$ : contravariant component in the $\xi$-direction

( $)^{\eta}$ : contravariant component in the $\eta$-direction

$\overline{()}$ : mean value

\section{Governing Equations}

The system used in the numerical analysis is schematically depicted in Fig. 1. In the initial stationary state, the flow duct is filled with pressurized hexane-based magnetic fluid, and flow immediately occurs when the outlet D-C is opened. Magnetic fluid is continuously introduced with high speed via the inlet section A-B, the flow is accelerated at the point of the venturi channel, and the inception of cavitation is induced by a pressure decrease.

In the present numerical formulation of the cavitating flow characteristics of magnetic fluid, we extend the general two-fluid model to the new vapor-liquid multiphase fluid model taking into account the effect of strong nonuniform magnetic field for analysis, which is based on the unsteady thermal nonequilibrium two-fluid model of Kataoka $^{(10)}$, and Harlow and Amsden ${ }^{(11)}$. In the numeri- 


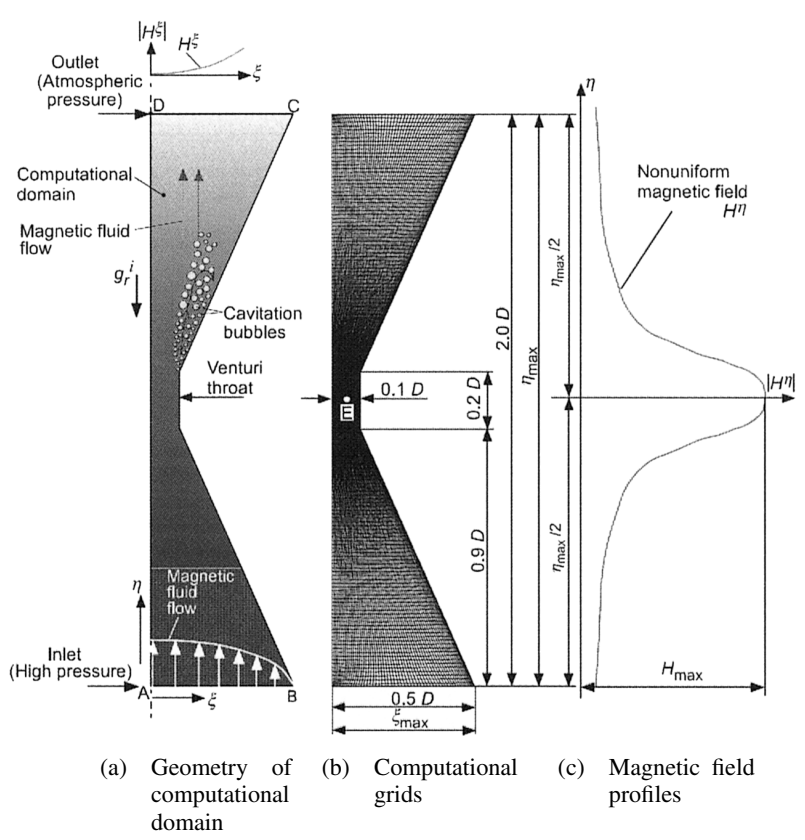

Fig. 1 Schematic of computational system used in numerical analysis

cal model, the condition of the working fluid with the cavitating magnetic fluid flow structure can be approximated to form a homogeneously dispersed bubbly flow, because the physical properties such as latent heat for vaporization, density, viscosity and surface tension of the hexanebased magnetic fluid are very small compared with that of the pure water at room temperature. Accordingly, it seems reasonable to assume that the cavitating flow pattern is easily formed in the bubbly two-phase flow. In the process of modeling, to consider the effects of the rapid evaporation and condensation of magnetic fluid, we apply the rapid phase change model of Yamamoto et al. ${ }^{(12)}$ and Young ${ }^{(13)}$ to the cavitating flow of magnetic fluid.

The calculation is carried out using the twodimensional generalized curvilinear coordinate system $(\xi, \eta)$ as shown in Fig. $1 ; \xi$ and $\eta$ denote the transverse and longitudinal coordinate, respectively. The model for analysis simulates the cavitating flow of magnetic fluid passing through the venturi channel in a vertical duct. It is assumed that the flow field is homogeneous in the $\eta$-direction and is symmetric to the central axis D-A as shown in Fig. 1. The nonuniform magnetic field is applied in the longitudinal $\eta$-direction, which is parallel to the mainstream of working fluid flow. In the numerical modeling under this condition, the following assumptions are employed to formulate the governing equations.

(1) The cavitating flow is a two-dimensional unsteady internal flow.

(2) The magnetic field is not influenced by the existence of the gas phase.

(3) The applied magnetic field is homogeneous and steady.
(4) The energy exchange between the liquid and gas phases is taken into account.

( 5 ) The liquid phase is incompressible fluid.

Under the above conditions, the governing equations of the cavitating magnetic fluid flow, taking into account the effect of nonuniform magnetic field based on the unsteady two-dimensional two-fluid model, are derived as follows.

The mass conservation equation for a gas and liquid phases is

$$
\frac{\partial}{\partial t}\left(\alpha_{m} \rho_{m}\right)+\nabla_{j}\left(\alpha_{m} \rho_{m} u_{m}^{j}\right)=\Gamma_{m},
$$

where the subscript $m$ denotes the gas phase $(m=g)$ or liquid phase $(m=l) . t$ is the time, $\alpha_{g}$ and $\alpha_{l}$ are the gas and liquid-phase volume fraction, respectively, $\rho_{g}$ and $\rho_{l}$ are the gas and liquid-phase density, respectively. The relationship $\left(\alpha_{g}+\alpha_{l}=1\right)$ is assumed. $u_{g}^{j}$ and $u_{l}^{j}$ are the gas and liquid-phase contravariant velocity, respectively, $\Gamma_{g}$ and $\Gamma_{l}$ are the gas and liquid-phase generation density, respectively, $\nabla_{j}$ is the covariant differential. The superscripts and subscripts $(i, j, k)$ denote the contravariant and covariant components, respectively, and the subscripts $g$ and $l$ denote the gas and liquid phases, respectively.

The combined equation of motion for a total gas and liquid phase is

$$
\begin{aligned}
& \frac{\partial}{\partial t}\left(\alpha_{g} \rho_{g} u_{g}^{i}+\alpha_{l} \rho_{l} u_{l}^{i}\right)+\nabla_{j}\left(\alpha_{g} \rho_{g} u_{g}^{i} u_{g}^{j}+\alpha_{l} \rho_{l} u_{l}^{i} u_{l}^{j}\right) \\
& \quad=-g^{i j} \nabla_{j} p_{l}+\mu_{0} \alpha_{l} M^{j} \nabla_{j} H^{i}+\beta_{T} g^{j k} \nabla_{j} \nabla_{k} u_{l}^{i} \\
& \quad+\frac{1}{3}\left(\beta_{T} \nabla_{j} \nabla_{k} u_{l}^{k}\right) g^{i j}+\left(\alpha_{g} \rho_{g}+\alpha_{l} \rho_{l}\right) g_{r}^{i},
\end{aligned}
$$

where the second term of the right-hand side of Eq. (2) represents the magnetic body force term in two-phase flow, $H^{i}$ is the contravariant vector of magnetic field, $M^{j}$ is the contravariant vector of magnetization, $g^{i j}$ is the fundamental metric tensor, $p$ is the absolute pressure, $\mu_{0}$ is the permeability in vacuum, and $g_{r}^{i}$ is the contravariant vector of gravitational acceleration.

Additionally, $\beta_{T}$ in Eq. (2) denotes the viscosity of the two-phase mixture flow that includes small dispersed bubbles. $\beta_{T}$ was evaluated using the following formula for the viscosity of a suspension $^{(14),(15)}$ :

$$
\beta_{T}=\left[1-\left(\frac{\alpha_{g}}{0.680}\right)\right]^{-2} \cdot \beta_{l}, \quad\left(\alpha_{g}<0.5\right) .
$$

To consider the effects of additional forces that act on the bubbles and the effects of radial expansion of the bubbles, the equation of motion for the gas phase is here replaced with the translational motion of a single bubble ${ }^{(16)}$. Therefore, the Eulerian-Lagrangian twoway coupling model ${ }^{(17),(18)}$ is applied to predict the twodimensional cavitating flow characteristics.

The equation of motion for the gas phase is

$$
\frac{4}{3} \pi \rho_{g} R_{g}^{3} \frac{d u_{g}^{i}}{d t}=-F_{p}^{i}+F_{g}^{i}-F_{D}^{i}-F_{V M}^{i}-F_{B}^{i}+F_{L M}^{i}+F_{L S}^{i},
$$


where each additional force term is derived as follows:

$$
\begin{aligned}
& F_{p}^{i}=\frac{4}{3} \pi R_{g}^{3} g^{i j} \nabla_{j} p_{l} \\
& F_{g}^{i}=\frac{4}{3} \pi R_{g}^{3} \rho_{g} g_{r}^{i} \\
& F_{D}^{i}=\frac{1}{2} \rho_{l} C_{D}\left|u_{g}^{i}-u_{l}^{i}\right|\left(u_{g}^{i}-u_{l}^{i}\right) \pi R_{g}^{2} \\
& F_{V M}^{i}=C_{V M} \cdot \rho_{l} \frac{4}{3} \pi R_{g}^{3}\left[\frac{d}{d t}\left(u_{g}^{i}-u_{l}^{i}\right)+\frac{3}{R_{g}}\left(u_{g}^{i}-u_{l}^{i}\right) \frac{d R_{g}}{d t}\right] \\
& F_{B}^{i}=6 R_{g}^{2} \sqrt{\pi \rho_{l} \beta_{l}} \int_{0}^{t} \frac{d}{d \tau}\left(u_{g}^{i}-u_{l}^{i}\right) \\
& F_{L M}^{i}=\pi R_{g}^{3} \rho_{l} e^{i j k}\left(\Omega_{g j}-\Omega_{l j}\right)\left(u_{g k}-u_{l k}\right) \\
& F_{L S}^{i}=6.46 \frac{\beta_{l} R_{g}^{2}}{\sqrt{\left|\left(\Omega_{g}^{i}-\Omega_{l}^{i}\right)\right| v_{l}}} e^{i j k}\left(\Omega_{g j}-\Omega_{l j}\right)\left(u_{g k}-u_{l k}\right) \\
& \Omega_{l}^{i}=\frac{1}{2} \omega_{l}^{i}=\frac{1}{4} e^{i j k}\left(\nabla_{j} u_{l k}-\nabla_{k} u_{l j}\right),
\end{aligned}
$$

where $F_{P}^{i}$ is the force due to the liquid phase pressure gradient, $F_{q}^{i}$ is the gravitational acceleration force, $F_{D}^{i}$ is the drag force, $F_{V M}^{i}$ is the virtual mass force considering the expansion of a bubble, and $F_{B}^{i}$ is the Basset history term which takes into account the effect of the deviation in flow pattern from the steady state. $F_{L M}^{i}$ is the Magnus lift force caused by the rotation of the bubble as reported by Auton et al. ${ }^{(19)} F_{L S}^{i}$ is Saffman's lift force ${ }^{(20)}$ caused by the velocity gradient of the liquid phase. $C_{D}$ is the drag coefficient, $C_{V M}$ is the virtual mass coefficient, $R_{g}$ is the equivalent bubble diameter, $\Omega^{i}$ is the contravariant angular velocity, and $\omega^{i}$ is the contravariant vorticity. $d / d t$ denotes the substantial derivative.

The equation for the angular velocity of a bubble is derived as follows ${ }^{(20)}$ :

$$
\frac{d \Omega_{g}^{i}}{d t}=\frac{15 \beta_{l}}{R_{g}^{2} \cdot \rho_{g}}\left(\Omega_{l}^{i}-\Omega_{g}^{i}\right) .
$$
is

The energy equation for the gas and the liquid phases

$$
\begin{aligned}
& \frac{\partial}{\partial t}\left(\alpha_{m} \rho_{m} e_{m}\right)+\nabla_{j}\left(\alpha_{m} \rho_{m} e_{m} u_{m}^{j}\right) \\
& \quad=-p_{m} \frac{\partial \alpha_{m}}{\partial t}-\nabla_{j}\left(\alpha_{m} p_{m} u_{m}^{j}\right) \\
& \quad+\Gamma_{m} h_{m}^{(i)}+q_{m}^{(i)} a^{(\mathrm{i})}-\nabla_{j}\left(\alpha_{m} q_{m}^{j}\right)+\alpha_{m} \Phi_{m} .
\end{aligned}
$$

In the above equation, the subscript $m$ denotes the gas phase $(m=g)$ or liquid phase $(m=l) . h_{g}^{(i)}$ and $h_{l}^{(i)}$ are the enthalpy of the gas phase and the liquid phase at the interface, respectively. $a^{(\mathrm{i})}$ is the gas-liquid interfacial area concentration per unit volume. $\Gamma_{g} h_{g}^{(i)}$ and $\Gamma_{l} h_{l}^{(i)}$ are the interfacial energy transfer terms due to the liquid-vapor phase change. $q_{g}^{(i)}$ and $q_{l}^{(i)}$ are the heat transfer terms of mutual interaction between the vapor and liquid interface. $q^{j}$ is the contravariant heat flow vector and $\Phi$ is the energy dissipation function, as described below:

$$
\left\{\begin{array}{l}
q_{m}^{i}=-\lambda_{m} g^{i j} \nabla_{j} T_{m}, \\
\Phi_{m}=-\frac{2}{3} \beta_{m}\left(\nabla_{i} u_{m}^{i}\right)^{2}+2 \beta_{m} s_{j m}^{i} s_{i m}^{j}, \\
s_{j m}^{i}=\frac{1}{2}\left(\nabla_{j} u_{m}^{i}+\nabla_{i} u_{m}^{j}\right) .
\end{array}\right.
$$

Assuming that the mass of each vapor bubble and of the condensed liquid droplet in each computational location is constant results in the following mass conservation equation for number density, $N_{k}$ :

$$
\begin{aligned}
& \frac{\partial}{\partial t}\left(\frac{4}{3} \pi R_{k}^{3} N_{k} \rho_{k}\right)+\nabla_{j}\left(\frac{4}{3} \pi R_{k}^{3} N_{k} \rho_{k} u_{k}^{j}\right)=\Gamma_{k}, \\
& \left\{\begin{array}{l}
k=e: R_{k}=R_{g}, N_{k}=N_{g}, \rho_{k}=\rho_{g}, u_{k}^{i}=u_{g}^{i}, \Gamma_{k}=\Gamma_{g} \\
k=c: R_{k}=R_{l}, N_{k}=N_{l}, \rho_{k}=\rho_{l}, u_{k}^{i}=u_{l}^{i}, \Gamma_{k}=\Gamma_{l},
\end{array}\right.
\end{aligned}
$$

where subscript $k$ denotes evaporation $(k=e)$ or condensation $(k=c)$.

The governing equations of cavitating flow mentioned above are constructed by Eulerian-type equations for the liquid phase and by Lagrangian-type equations for the gas phase.

\section{1 Constitutive equations}

The drag coefficient, $C_{D}$, and the virtual mass coefficient, $C_{V M}$, are defined as follows ${ }^{(20)}$ :

$$
\left\{\begin{array}{l}
C_{D}=\frac{24}{R e_{B}}\left(1+0.15 \operatorname{Re}_{B}{ }^{0.687}\right)+\frac{0.42}{1+42500 \operatorname{Re}_{B}{ }^{-1.16}} \\
C_{V M}=0.5 \\
\operatorname{Re}_{B}=\frac{\rho_{l}\left|u_{g}^{i}-u_{l}^{i}\right| D}{\beta_{l}}
\end{array}\right.
$$

The energy balance condition at the interface of the gas and liquid phases is expressed by the following equation:

$$
\left\{\begin{array}{l}
\Gamma_{g} h_{g}^{(i)}+\Gamma_{l} h_{l}^{(i)}=0 \\
q_{g}^{(i)}+q_{l}^{(i)}=0
\end{array}\right.
$$

where $h_{k}=c_{p k} T_{k} ;(k=g, l)$. The detailed constitutive equations for interfacial energy transfer terms in Eq. (18) are given by following extended empirical formulas ${ }^{(21),(22)}$ :

$$
q_{g}^{(i)}=k^{(i)}\left(T_{g}-T_{s}\right),
$$

where $k^{(i)}$ is the interfacial heat transfer rate between the gas and liquid phases, and is given by following equations $^{(21)}$ :

$$
\left\{\begin{array}{l}
k^{(i)}=\alpha k_{g}^{(i)}+\alpha_{l} k_{l}^{(i)} \\
k_{g}^{(i)}=\frac{8.067 \cdot \lambda_{g}}{R_{g}} \\
k_{l}^{(i)}=\frac{1.0+0.37 \operatorname{Re}_{V}{ }^{0.50} \cdot \operatorname{Pr}_{V}{ }^{0.35}}{R_{g}} \\
\operatorname{Re}_{V}=\frac{2.0 R_{g}\left|u_{g}^{i}-u_{l}^{i}\right|}{v_{l}} \\
\operatorname{Pr}_{V}=\frac{c_{p l} \cdot \beta_{l}}{\lambda_{l}} .
\end{array}\right.
$$


It is assumed that the energy transfer is caused by the heat transfer between the isothermal spherical bubble and the surrounding liquid. Assuming a spherical bubble with equivalent radius $R_{g}$, the expression of the interfacial area concentration per unit volume $a^{(\mathrm{i})}$ is obtained by the following equation ${ }^{(10)}$ :

$$
a^{(\mathrm{i})}=\frac{3 \alpha_{g}}{R_{g}} .
$$

In general, the interfacial transfer terms are proportional to the interfacial area concentration, $a^{(\mathrm{i})}$. Therefore, $a^{(\mathrm{i})}$ is one of the most important parameters in the two-fluid model. Assuming that the vapor gas phase follows an ideal gas law and that the relationship between gas phase pressure, $p_{g}$, and density, $\rho_{g}$, obeys polytropic change, the following equation by Hirt and Romero ${ }^{(23)}$ results:

$$
\begin{aligned}
& \rho_{g}\left(\kappa_{g}-1\right) e_{g}=\left[p_{g}-c_{l 0}^{2} \rho_{l}\left(\alpha_{g}^{*}-\alpha_{g}\right)\right] \alpha_{g}^{*} \\
& \begin{cases}\alpha_{g} \geq \alpha_{g c}: & \alpha_{g}^{*}=\alpha_{g}, \\
\alpha_{g}<\alpha_{g c}: & \alpha_{g}^{*}=\alpha_{g c},\end{cases}
\end{aligned}
$$

where $c_{l 0}$ is the sound velocity in the hexane-base magnetic fluid at the initial state $\left(c_{l 0}=1050.0 \mathrm{~m} / \mathrm{s}\right)$, and $\alpha_{g c}$ denotes the threshold of the void fraction $\left(\alpha_{g c}=0.005\right)^{(23)}$.

The constitutive equation for gas phase generation density, $\Gamma_{g}$, is defined by the following equation:

$$
\Gamma_{g}=\Gamma_{g e}-\Gamma_{g c},
$$

where $\Gamma_{g e}$ and $\Gamma_{g c}$ denote the gas phase evaporation density and gas phase condensation density, respectively. By introducing constitutive equations for $\Gamma_{g e}$ and $\Gamma_{g c}$ to consider the effect of the surface tension $\left(\gamma_{l}\right)$ in the cavitation inception process, we extend the classical nucleation theory for water droplets from subcooled vapor to the hexanebased magnetic fluid. Namely, $\Gamma_{g e}$ and $\Gamma_{g c}$ are assumed to be proportional to the degree of subcooling and superheat. Furthermore, if $\Gamma_{g k}(k=e, c)$ is expressed by the sum of the nucleation rate of the evaporated bubble or the condensed liquid droplet, and is also expressed by the increase in mass due to the growth of vapor bubbles and condensed droplets, the following equations for $\Gamma_{g k}$ are derived $^{(12),(13)}$ :

$$
\begin{aligned}
& \Gamma_{g k}=\frac{4}{3} \pi \rho_{k} I_{k} R_{k(\mathrm{cr})}^{3}+4 \pi \rho_{k} \sum_{i=1}^{i_{\max }} N_{k i} R_{k i}^{2} \frac{d R_{k i}}{d t} \\
& \left\{\begin{array}{l}
I_{k}=\frac{A_{c}}{1+\Theta}\left(\frac{2 \gamma_{l}}{\pi m_{a}^{3}}\right)^{1 / 2} \frac{\rho_{g}^{2}}{\rho_{l}} \exp \left(-\frac{4 \pi R_{k(\mathrm{cr})}^{2} \gamma_{l}}{3 k_{B} T_{k}}\right) \\
\Theta=\frac{2\left(\kappa_{g}-1\right)}{\kappa_{g}+1} \frac{\Delta h}{\mathfrak{R} T_{g}}\left(\frac{\Delta h}{\mathfrak{R} T_{g}}-0.5\right) \\
R_{k(\mathrm{cr})} \approx \frac{2 \gamma_{l} T_{s}}{\rho_{k} \Delta h \Delta T} .
\end{array}\right.
\end{aligned}
$$

In Eq. (24), subscript $k$ has the same definition as that used in Eq. (16), $R_{k}$ is the radius of a bubble or droplet, $R_{k \text { (cr) }}$ is the Kelvin-Helmholtz critical nucleate radius, $k_{B}$ is Boltzmann's constant, $I_{k}$ is the nuclei generation rate of vapor bubbles or liquid droplets, $A_{c}$ is the condensation coefficient, $\Theta$ is the nonisothermal correction factor, $m_{a}$ is the mass of a single molecule of hexane, $\gamma$ is the surface tension, $\mathfrak{R}$ is the gas constant, $T_{s}$ is the saturation temperature, and subscript $i$ is the value at each calculation cell. $\Delta h$ denotes the latent heat which is described by the difference in specific enthalpy between the liquid and gas phases and is defined as $\Delta h=h_{l}-h_{g}$. The temperature difference between saturation temperature and gas- or liquidphase temperature, $\Delta T$, is defined as $\Delta T=\left|T_{s}-T_{k}\right| . N_{k i}$ denotes the number density of the generated vapor bubbles or condensed liquid droplets at each calculation cell, $i$.

By introducing the formulation of the growth process for bubbles and condensed droplets, we assume that the growth rate of a bubble or droplet is controlled by the rate at which the enthalpy of vaporization or condensation can be conducted away from the bubble and droplets to the bulk liquid ${ }^{(24)}$. Under that assumption, the equation of the growth process for a single vapor bubble and a condensed droplet is derived as

$$
\Delta h \rho_{k} \frac{d R_{k i}}{d t}=\frac{p_{k}}{\sqrt{2 \pi \mathfrak{R} T_{g}}} \frac{\kappa_{k}+1}{2 \kappa_{k}} c_{p k} \Delta T^{(i)},
$$

where $\Delta T^{(i)}$ denotes the interfacial temperature between the vapor phase and the condensed droplet and is derived by the following equation:

$$
\Delta T^{(i)}=\left(1-\frac{R_{k(\mathrm{cr})}}{R_{k i}}\right)\left|T_{s}-T_{k}\right| .
$$

\section{2 Numerical conditions and procedure}

As a practical example, we use the fluid properties of the a hexane-based temperature sensitive magnetic fluid with manganese-zinc ferrite particles of $50 \%$ weight concentration $^{(2)}$. The strength of magnetization $M$ is approximated by the previous measurement data ${ }^{(2)}$, and is expressed as a function of temperature $T_{l}$ and the strength of magnetic field $H$ as

$$
\left\{\begin{array}{l}
M=C_{a} \cdot f_{1}(H) \cdot f_{2}\left(T_{l}\right) \\
f_{1}(H)=290.0+0.171 H-6.809 \times 10^{-7} H^{2} \\
\quad+1.007 \times 10^{-12} H^{3} \\
\quad(H \leq 115.3 \mathrm{kA} / \mathrm{m}) \\
f_{2}\left(T_{l}\right)=6.675 \times 10^{4}-130.07 T_{l} \\
C_{a}=3.108 \times 10^{-5} .
\end{array}\right.
$$

Next, in order to consider the effect of nonuniform magnetic field as depicted in Fig. 1, the distributions of the longitudinal magnetic field component $H^{\eta}$ and the transverse magnetic field component $H^{\xi}$ are derived by the following equations. In introducing $H^{\eta}$ and $H^{\xi}$, we referred to the analytical solution of the nonuniform magnetic field distribution of Helmholtz coil ${ }^{(25)}$ and the measurement results of the magnetic field of the electromagnet which was used in the previous experimental study ${ }^{(1),(2)}$. 


$$
\left\{\begin{array}{l}
H^{\eta}=H_{\max } \cdot \exp \left(-\eta_{h}^{* 2}\right), \\
H^{\xi}=H_{\max } \cdot \xi^{*} \cdot\left|\eta_{h}^{*}\right| \cdot \exp \left(-\eta_{h}^{* 2}\right), \\
\eta_{h}^{*}=\eta^{*}-\frac{1}{2} \eta_{\max }^{*}
\end{array}\right.
$$

where $H_{\max }$ is the maximum magnetic field strength, $\eta^{*}$ denotes the normalized longitudinal coordinate which is defined as $\eta^{*}=\eta / \eta_{\max }$, and $\xi^{*}$ denotes the normalized transverse coordinate which is defined as $\xi^{*}=\xi / \xi_{\max }$. The $H_{\max }$ on the electromagnet is installed in the position of the throat of the venturi nozzle.

The finite difference method is used to solve the set of governing equations mentioned above. In the present calculation, the discrete forms of these equations are semiimplicitly obtained using a staggered grid. The grid is concentrated at the venturi nozzle wall to capture the cavitation inception precisely. Then the modified SOLA (numerical SOLution Algorithm for transient fluid flow) method of Tomiyama et al. ${ }^{(26)}$, which is superior for the formulation and solution of a gas liquid two-phase flow problem, is applied for the numerical calculation. The liquid phase velocity, $u_{l}^{i}$, at the location of bubbles is calculated using an area-weighting interpolation method which is used in the SMAC algorithm by Amsden and Harlow ${ }^{(27)}$.

To determine the boundary conditions, free-slip condition for prescribed liquid phase velocity is applied to the central axis, D-A, nonslip condition for prescribed liquid phase velocity is applied to the sidewall, C-B, in Fig. 1. Also, a fully developed parabolic velocity profile is continuously applied for liquid phase velocities to the inlet cross-sectional area of the flow duct, A-B. A convective outflow condition is applied for liquid phase velocities to the exit section of the duct, D-C. Adiabatic conditions are applied for thermal boundary conditions at the duct wall surface. The initial stationary condition of the liquid phase is assumed to be the pressurized fluid state.

The conditions for numerical analysis are listed in following Table 1. For other physical properties used in constitutive equations, $\beta_{l}$ and $\lambda_{l}$ are given as a function of temperature and are defined by following empirical formula:

Table 1 Condition for numerical analysis

\begin{tabular}{lccc}
\hline Density & $\rho_{l}$ & 1386.0 & $\mathrm{~kg} / \mathrm{m}^{3}$ \\
Inlet pressure & $p_{l(\mathrm{in})}$ & 0.20 & $\mathrm{MPa}$ \\
Outlet pressure & $p_{l(\mathrm{ex})}$ & 0.101 & $\mathrm{MPa}$ \\
Inlet temperature & $T_{l(\mathrm{in})}$ & 283 & $\mathrm{~K}$ \\
Inlet internal energy & $e_{l(\mathrm{in})}$ & 1205.0 & $\mathrm{~kJ} / \mathrm{kg}$ \\
Surface tension & $\gamma_{l}$ & 0.0213 & $\mathrm{~N} / \mathrm{m}$ \\
Inner width of duct & $D$ & 8.0 & $\mathrm{~mm}$ \\
\hline
\end{tabular}

$$
\left\{\begin{aligned}
\beta_{l}= & \rho_{l}\left(2.247 \times 10^{-4}-1.976 \times 10^{-6} T_{l}\right. \\
& \left.+5.897 \times 10^{-9} T_{l}^{2}-5.926 \times 10^{-12} T_{l}^{3}\right) \quad[\mathrm{Pa} \cdot \mathrm{s}] \\
& \lambda_{l}=0.1412+3.4659 \times 10^{-4} T_{l}-2.086 \times 10^{-6} T_{l}^{2} \\
& +2.431 \times 10^{-9} T_{l}^{3} \quad[\mathrm{~W} /(\mathrm{m} \cdot \mathrm{K})]
\end{aligned}\right.
$$

The saturation temperature $T_{s}$ is given as a function of pressure. The other required physical properties of the liquid phase are given by the tables of the thermophysical properties of hexane ${ }^{(28)}$.

The interval of each time-step is automatically adjusted during the computation to satisfy the CFL condition $^{(15)}$. We actually calculated solutions on three different grid densities: $50 \times 120,60 \times 150$ and $100 \times 220$ nodes. As a result, we found that the each numerical result shows almost the same profile, the grid independence of the numerical results was confirmed. Thus, as a compromise between computer memory and accuracy, we chose to use the $60 \times 150$ structured grid in the $\xi$ and $\eta$-directions for the calculations. While the execution of the unsteady calculation, no significant differences in the mean flow profiles are found in the 1200 to 1500 time-steps. We determined that the cavitating flow almost reaches steady state when such flow profiles are obtained.

\section{Results and Discussion}

Figure 2 shows the numerical results of the transient evolution of the void fraction $\left(\alpha_{g}\right)$ contour, and Fig. 3 shows the instantaneous liquid phase pressure $\left(p_{l}\right)$ contour. According to Fig. 2, the cavitation inception effectively occurs at the position of the divergent venturi throat. In the case of strong magnetic field $\left(H_{\max }=115.3 \mathrm{kA} / \mathrm{m}\right)$, cavitation formation of cloud cavity at the diverging venturi throat is suppressed compared with that of nonmagnetic field $\left(H_{\max }=0 \mathrm{kA} / \mathrm{m}\right)$ due to the magnetic body force under a sharp magnetic field gradient. It was found that the growth rate of the volume fraction of cavitation bubble in strong magnetic field is smaller than that in nonmagnetic field. Immediately after the flow is initially induced, taking note of the primary feature of the void fraction profile under strong magnetic field, the void fraction profile elongated in the longitudinal direction of negative magnetic field gradient because the bubbles are accelerated and migrate due to the two-phase magnetic body force in the direction of the negative magnetic field gradient. The effect of two-phase magnetic body force is characterized by the second right-hand term in the momentum equation, Eq. (2).

With time elapses, because the magnetic body force based on the transverse magnetic field gradient acts so that the bubbles migrate from the wall into the center of the duct, it becomes clear that the void fraction is locally increasing near the central axis. Downstream of the venturi throat, although magnetic body force acts as a flow resis- 


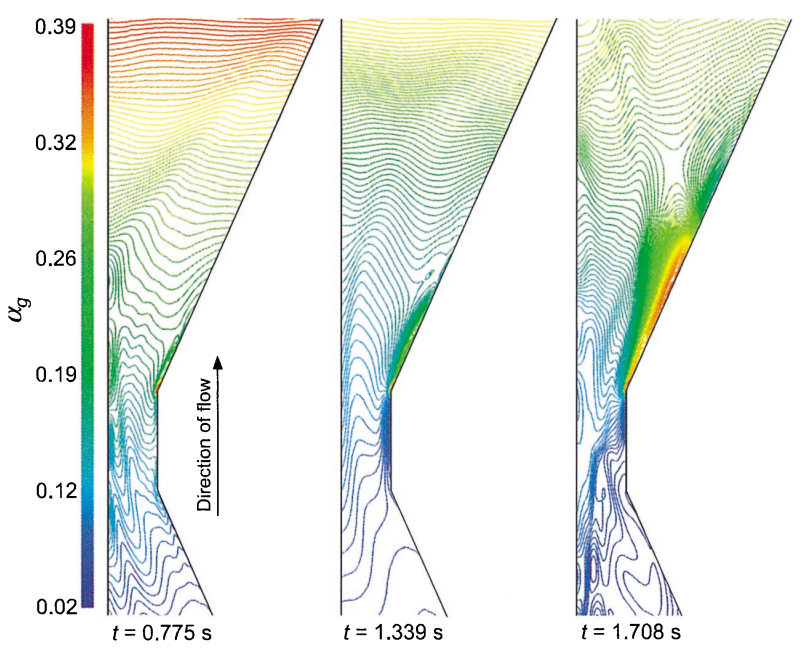

(a) $H_{\max }=115.3 \mathrm{kA} / \mathrm{m}$

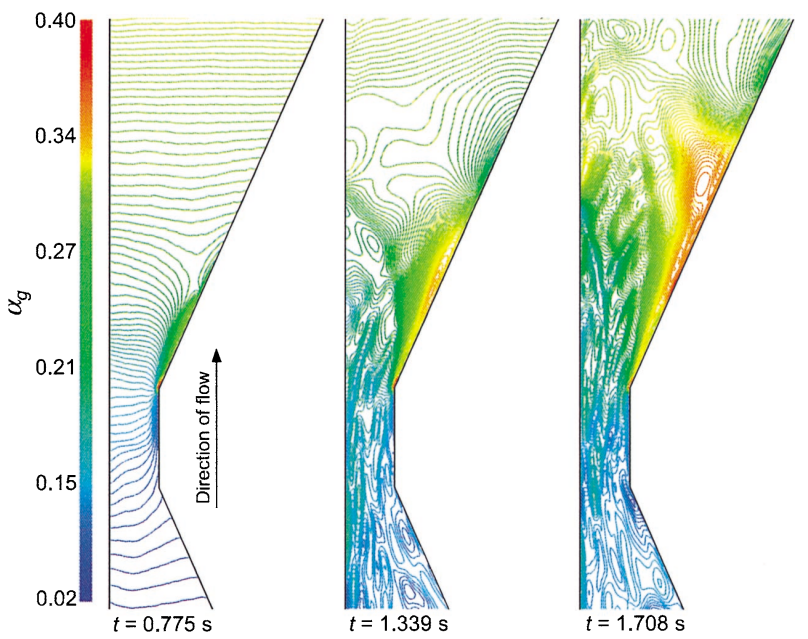

(b) In nonmagnetic field

Fig. 2 Effect of nonuniform magnetic field on time evolution of void fraction distributions (Enlarged view of venturi throat section)

tance, the forces are decreased due to the gas phase inclusion. In the two-phase flow region, not only the decreasing effect of flow resistance due to the negative magnetic field gradient, but also the pressure rise effect caused by the unbalance of the magnetic body force between single- and two-phase flow region is obtained.

Furthermore, additional lift force operates in the direction which causes the bubbles to migrate in the duct wall direction. However, as the two-phase magnetic body force begins to dominate the bubbles, it is found that the large volume fraction region of gas phase moves to the central axis of the duct. According to this result, the gas phase motion is controlled not only by the buoyancy force but also due to the liquid phase pressure gradient, additional lift forces, and especially due to the two-phase magnetic body force that acts on the cavitation bubbles.

If the phenomenon applicable to the conditions of both strong magnetic field and nonmagnetic field is as ex-

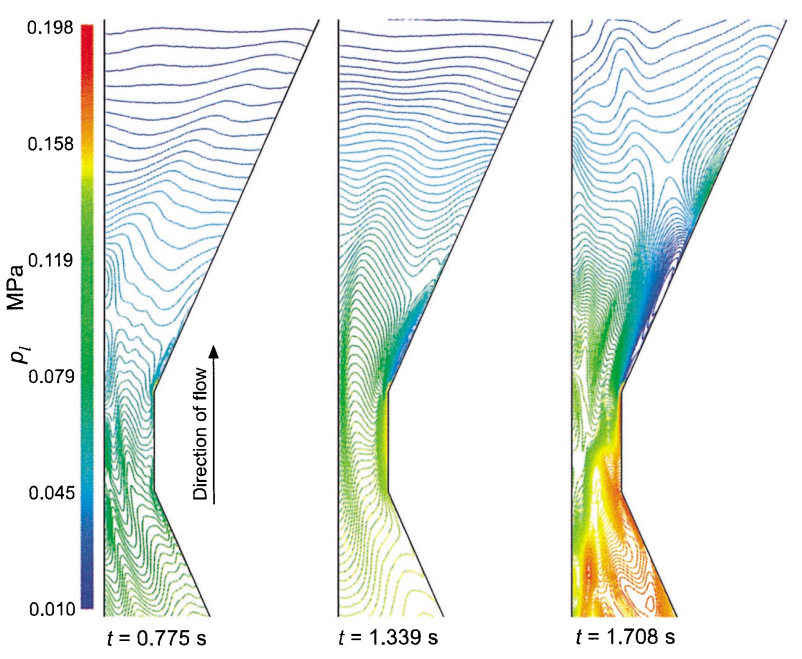

(a) $H_{\max }=115.3 \mathrm{kA} / \mathrm{m}$

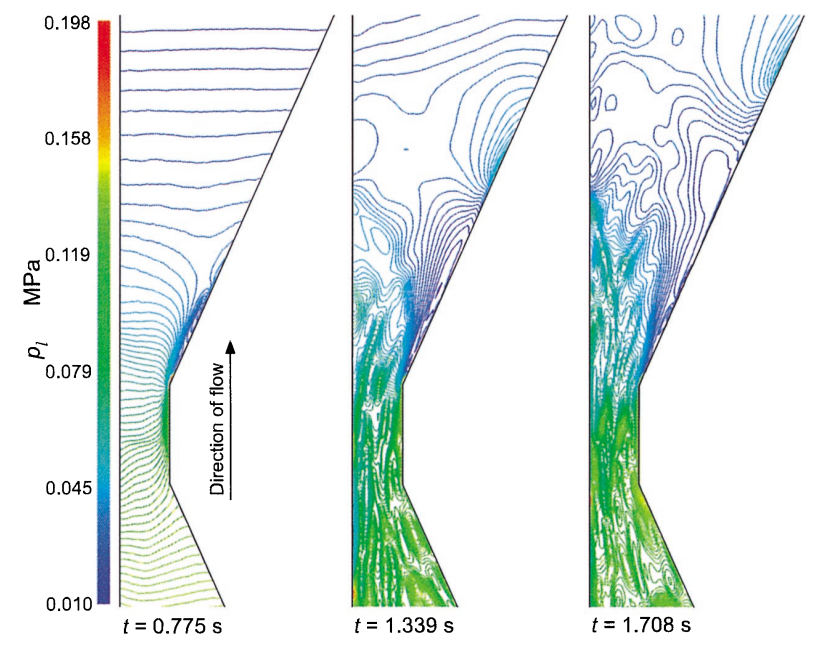

(b) In nonmagnetic field

Fig. 3 Effect of nonuniform magnetic field on instantaneous liquid phase pressure contours (Enlarged view of venturi throat section)

plained here, the gas phase volume fraction increases at the throat position and is concentrated to form a small cavity cloud downstream of divergent venturi nozzle due to the small vortex induced by the wake passing through the venturi throat, based on the effect of the negative pressure gradient. When the magnitude of the cloud cavity is above a certain size, due to the re-entrant jet resulting from the boundary layer separation, the cavity becomes detached from the cloud and then remains in the high volume fraction region as the gas phase moves downstream. Especially in the case of strong magnetic field, because the twophase magnetic ejection effect acts on the cloud cavity in the negative magnetic field gradient region, the separation of the cloud cavity is enhanced by the two-phase magnetic body force acting on the cavitation bubbles. Due to the suppression effect and magnetic ejection effect on the cavitation bubbles, and the enhancement of the cloud cavity separation, the magnitude of the cloud cavity under strong 
magnetic field becomes smaller than that of nonmagnetic field, and the more homogeneous two-phase flow is generated under strong magnetic field compare to the case of nonmagnetic field.

Figure 4 shows the present numerical results of the liquid phase pressure rise effect of in the longitudinal direction, in comparison with the previous one-dimensional numerical result of its effect for the steady boiling twophase pipe flow of magnetic fluid, and the experimental results of pressure rise effect along longitudinal direction for steady boiling two phase pipe flow of magnetic fluid ${ }^{(2)}$. In this figure, the axis of the ordinate denotes the normalized pressure rise parameter, $\Delta p_{l}^{*}$, and is derived by the following equation.

$$
\Delta p_{l}^{*}=\frac{\overline{p_{l(\mathrm{eff})}}-p_{l(\mathrm{in})}}{p_{l(\mathrm{in})}},
$$

where $\overline{p_{l(\text { eff })}}$ is the cross-sectional mean effective driving pressure from which the influence on the prudence of the liquid phase fluid can be deducted. $p_{l(\text { in })}$ is the inlet pressure. The axis of the abscissa in Fig. 4 denotes the normalized longitudinal coordinate $\eta^{*}\left(=\eta / \eta_{\max }\right)$. Focusing on the present numerical result of cavitating magnetic fluid flow, the pressure rise effect based on the two-phase magnetic body force under an applied magnetic field region acts effectively at the initial stage of cavitating flow in the two-phase downstream region. However, as time elapses and the flow approaches steady state, it is found that the pressure rise effect, which is based on cavitating two-phase magnetic body force, decreases around the exit section of the duct.

Comparing our previous results regarding the pres-

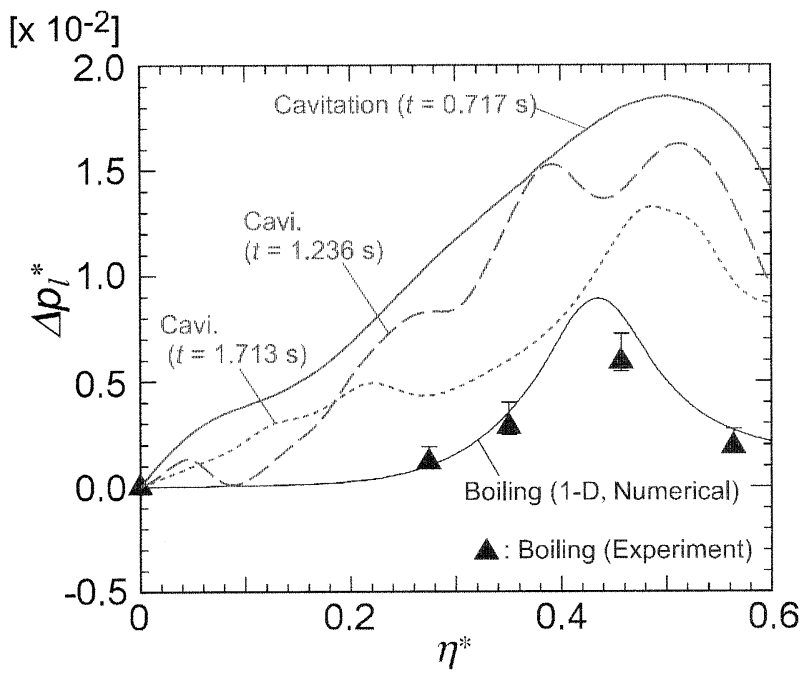

Fig. 4 Normalized pressure rise effect $\Delta p_{l}^{*}$ in cavitating flow of magnetic fluid along the central axis A-D, in comparison with the previous one-dimensional numerical result and experimental results of $\Delta p_{l}^{*}$ along the longitudinal direction for steady boiling two-phase pipe flow of magnetic fluid $^{(2)}$ sure distribution of the boiling two-phase magnetic fluid flow $^{(2)}$ with the present numerical results, it is found that the present fluid acceleration system which uses cavitation can achieve a greater pressure rise effect. Therefore, when the fluid driving system which uses two-phase magnetic fluid is employed, rather high pressure rise effect resulting from the use of cavitation for two-phase flow production can be obtained.

Figure 5 shows the effect of magnetic acceleration on the longitudinal liquid phase cross-sectional mean velocity, $\overline{u_{l}^{\eta}}$, as a function of time, $t$. In the case of strong magnetic field (a), the fluid acceleration effect which is based on the two-phase magnetic body force, increases with the lapse of time. It is found that $\overline{u_{l}^{\eta}}$ sharply increases in the flow field becomes two-phase flow state, especially

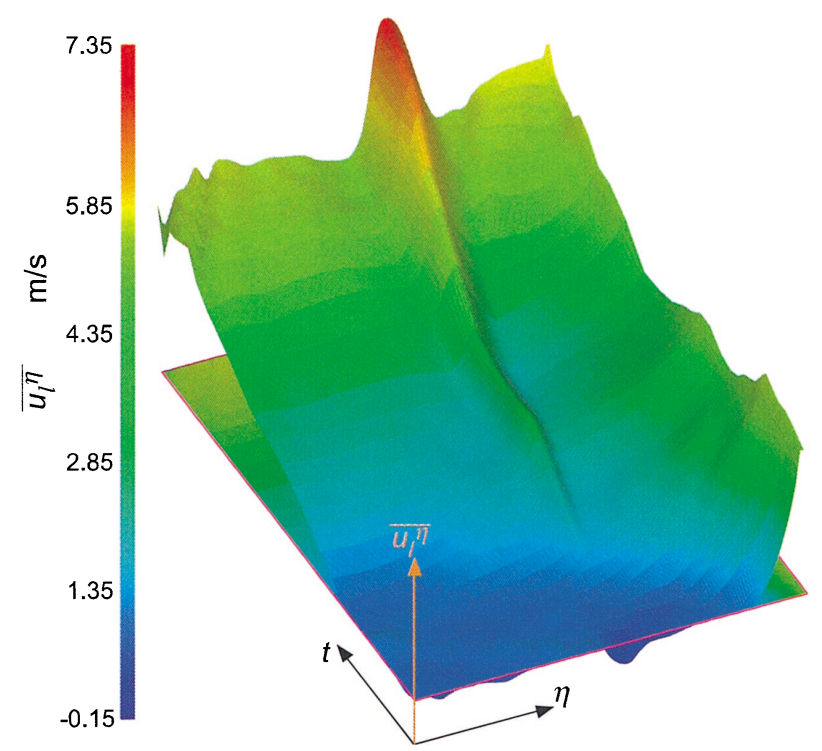

(a) $H_{\max }=115.3 \mathrm{kA} / \mathrm{m}$

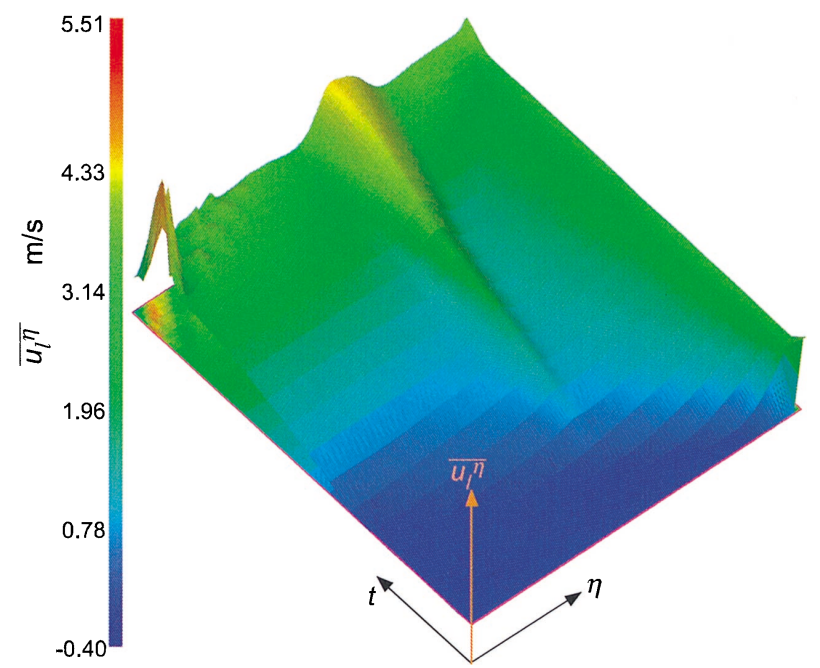

(b) In nonmagnetic field

Fig. 5 Effect of nonuniform magnetic field on longitudinal liquid phase velocity component along the central axis $\mathrm{D}-\mathrm{A}$ as a function of time 
increases at the converging section of the venturi throat ( $\eta=0.5 \eta_{\max }$ ), and that $\overline{u_{l}^{\eta}}$ tends to express the maximum value at the exit section of the duct. In the case of nonmagnetic field (b), it is found that $\overline{u_{l}^{\eta}}$ increases with increase in time, $t$. However, the magnitude of $\overline{u_{l}^{\eta}}$ exhibits a lower value than that of $\overline{u_{l}^{\eta}}$ in the case of strong magnetic field. Furthermore, it is found that the rate of increase on $\overline{u_{l}^{\eta}}$ in strong magnetic field shows a greater value than that of nonmagnetic field.

Figure 6 shows the profiles of the liquid phase velocity $\left(u_{l}^{i}\right)$ around the throat section. The flow separation and backward-flow of $u_{l}^{i}$ locally occur in the vicinity of the wall of the throat section upstream of the point of cavitation inception. In the case of strong magnetic field $\left(H_{\max }=115.3 \mathrm{kA} / \mathrm{m}\right)$, the liquid phase is locally accelerated around the throat wall section and the magnitude of $u_{l}^{i}$ has a larger value compared with the case of nonmagnetic field because of the two-phase magnetic acceleration effect which acts on the liquid phase due to the
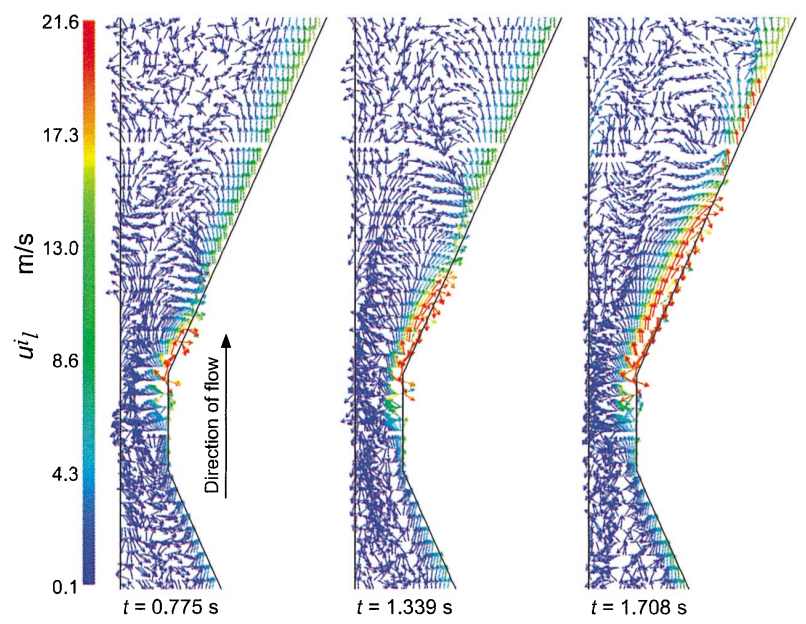

(a) $H_{\max }=115.3 \mathrm{kA} / \mathrm{m}$

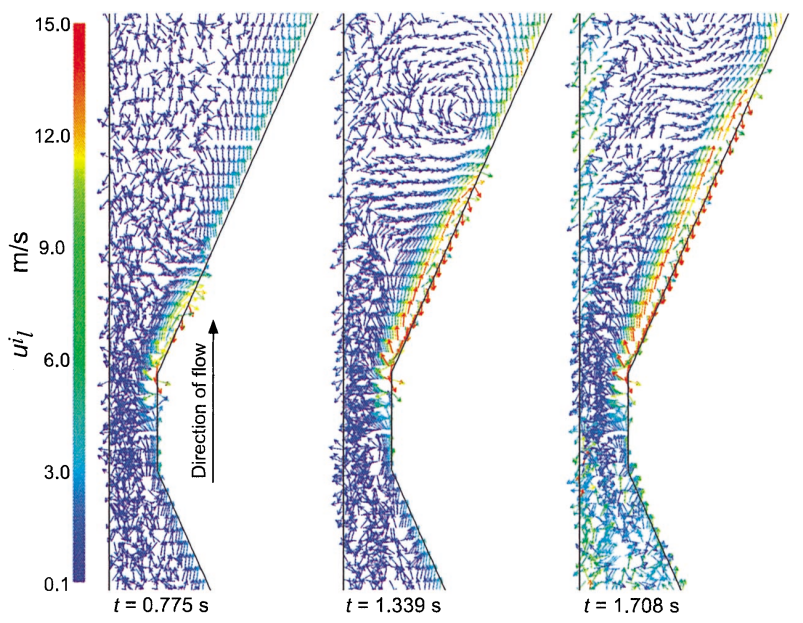

(b) In nonmagnetic field

Fig. 6 Effect of nonuniform magnetic field on instantaneous liquid phase velocity vector (Enlarged view of venturi throat section) strong magnetic field gradient. In the throat wall vicinity where cavitation actively generates, the liquid phase velocity is strongly accelerated because of the two-phase magnetic driving force and bubbles' pumping effect acting on the liquid phase. The slip ratio $\left(u_{g}^{i} / u_{l}^{i}\right)$ in the vicinity of throat wall section tend to have a large value. Furthermore, because of the increase in momentum exchange between the gas and liquid phases, the magnitude of $u_{l}^{i}$ locally increases in the region where the cavitation is actively generated. According to the results, it is found that rather than utilizing only the pumping effect of the bubbles when employing the two-phase fluid driving system, the method which utilizes the two-phase magnetic body force generated by cavitating magnetic fluid flow can obtain the enhanced fluid acceleration effect.

\section{Conclusions}

(1) Formations of the cavitation bubbles and the cloud cavity in the case of strong magnetic field in the diverging venturi throat is more greatly suppressed than in the case of nonmagnetic field due to the magnetic body force with a sharp magnetic field gradient. Especially in the case of strong magnetic field, because the two-phase magnetic ejection effect acts on the cloud cavity in the negative magnetic field gradient region, separation of the cloud cavity is enhanced by the two-phase magnetic body force acting on the cavitation bubbles.

(2) The pressure rise effect due to the two-phase magnetic body force under an applied magnetic field region effectively acts in the downstream two-phase region. The magnitude of the pressure rise effect in the cavitating magnetic fluid flow has a larger value than boiling twophase magnetic fluid flow.

(3) It is found that rather than utilizing only the pumping effect of bubbles when employing the two-phase fluid driving system, the method which utilizes the twophase magnetic body force generated by cavitating magnetic fluid flow can obtain the enhanced fluid acceleration effect.

\section{Acknowledgements}

The authors would like to thank Prof. Hideya Nishiyama (Inst. Fluid Sci., Tohoku University, Japan) for his helpful discussions. This research was supported by a Grant-in-Aid for Scientific Research (C. No. 15760099) from Ministry of Education, Culture, Sports, Science and Technology.

\section{References}

( 1 ) Ishimoto, J., Okubo, M., Nishiyama, H. and Kamiyama, S., Basic Study on an Energy Conversion System Using Gas-Liquid Two-Phase Flows of Magnetic Fluid (Analysis on the Mechanism of Pressure Rise), JSME Int. J., Ser. B, Vol.39, No.1 (1995), pp.7279. 
(2) Kamiyama, S. and Ishimoto, J., Boiling Two-Phase Flows of Magnetic Fluid in a Nonuniform Magnetic Field, J. Magn. Magn. Mat., Vol.149 (1995), pp.125131.

( 3 ) Petrick, M. and Branover, H., Liquid Metal MHD Power Generation-Its Evolution and Status, Prog. Astro. Aero., Vol.100 (1985), pp.371-400.

( 4 ) Charles, S.W. and Popplewell, J., Progress in the Development of Ferromagnetic Liquids, IEEE Trans. on Magnetics, Vol. MAG-16 (1980), pp.172-177.

( 5 ) Fedonenko, A.I. and Smirnov, V.I., Particle Interaction and Clumping an Electrically Conducting Magnetic Fluid, J. Magn. Magn. Mat., Vol.19 (1983), pp.388391.

(6) Okubo, M., Ishimoto, J., Nishiyama, H. and Kamiyama, S., Analytical Study on Two-Phase MHD Flow of Electrically Conducting Magnetic Fluid, Magnetohydrodynamics, Vol.29, No.3 (1983), pp.291-297.

( 7 ) Eckert, S., Gerbeth, G. and Lielausis, O., The Behaviour of Gas Bubbles in a Turbulent Liquid Metal Magnetohydrodynamic Flow, Part I: Dispersion in Quasi-Two-Dimensional Magnetohydrodynamic Turbulence, Int. J. Multiphase Flow, Vol.26, No.1 (2000), pp.45-66.

( 8 ) Eckert, S., Gerbeth, G. and Lielausis, O., The Behaviour of Gas Bubbles in a Turbulent Liquid Metal Magnetohydrodynamic Flow, Part II: Magnetic Field Influence on the Slip Ratio, Int. J. Multiphase Flow, Vol.26, No.1 (2000), pp.67-82.

(9) Ishimoto, J., Okubo, M. and Kamiyama, S., Effect of Magnetic Field on the Stability of Boiling Two-Phase Flows of Magnetic Fluid, Proc. 2nd Int. Conf. Multiphase Flow, Vol.4 (2001), pp.FC17-FC24.

(10) Kataoka, I. and Serizawa, A., Basic Equations of Turbulence in Gas-Liquid Two-Phase Flow, Int. J. Multiphase Flow, Vol.15, No.5 (1989), pp.843-855.

(11) Harlow, F.H. and Amsden, A.A., Numerical Calculation of Multiphase Fluid Flow, J. Comput. Phys., Vol.17, No.1 (1975), pp.19-52.

(12) Yamamoto, S., Hagari, H. and Murayama, M., Numerical Simulation of Condensation around the 3-D Wing, Trans. Jpn. Soc. Aero. Space Sci., Vol.42, No.138 (1997), pp.182-189.

(13) Young, J.B., Two-Dimensional, Nonequilibrium, WetStream Calculations for Nozzles and Turbine Cascades, Trans. ASME J. Turbomach., Vol.114 (1992), pp.569-579.

(14) Cross, M.M., Viscosity-Concentration-Shear Rate Relations for Suspensions, Rheologica Acta, Vol.14
(1975), pp.402-403.

(15) Tomiyama, A. and Shimada, N., A Numerical Method for Bubbly Flow Simulation Based on a Multi-Fluid Model, Trans. ASME J. Pressure Vessel Tech., Vol.123, No.4 (2001), pp.510-516.

(16) Fan, L.S. and Zhu, C., Principles of Gas-Solid Flows, (1998), Cambridge University Press, New York, NY.

(17) Patankar, N.A. and Joseph, D.D., Modeling and Numerical Simulation of Particle Flows by the EulerianLagrangian Approach, Int. J. Multiphase Flow, Vol.27, No.10 (2001), pp.1659-1684.

(18) Murai, Y. and Matsumoto, Y., Numerical Study of the Three-Dimensional Structure of a Bubble Plume, Trans. ASME J. Fluids Eng., Vol.122, No.4 (2000), pp.754-760.

(19) Auton, T.R., The Force Exerted on a Body in Invisid Unsteady Non-Uniform Rotational Flow, J. Fluid Mech., Vol.197 (1988), pp.241-257.

(20) Clift, R., Grace, J.R. and Weber, M.E., Bubbles, Drops, and Particles, (1978), Academic Press, San Diego, CA.

(21) Dobran, F., Liquid and Gas-Phase Distributions in a Jet with Phase Change, Trans. ASME J. Heat Transf., Vol.110 (1988), pp.955-960.

(22) Solbrig, C.W., McFadden, J.H., Lyczkowski, R.W. and Hughes, E.D., Heat Transfer and Friction Correlations Required to Describe Steam-Water Behavior in Nuclear Safety Studies, AIChE Sympo. Ser., Vol.174 (1978), pp.100-128.

(23) Hirt, C.W. and Romero, N.C., Application of a Drift Flux Model to Flashing in Straight Pipes, LA-6005MS, Los Alamos Scientific Laboratory Report, (1975).

(24) Moses, C.A. and Stein, G.D., On the Growth of Steam Droplets Formed in Laval Nozzle Using Both Static Pressure and Light Scattering Measurements, Trans. ASME J. Fluids Eng., Vol.100 (1978), pp.311-322.

(25) Otis, D.R., Computation and Measurement of Hall Potentials and Flow-Field Perturbations in Magnetogasdynamic Flow of an Axisymmetric Free Jet, J. Fluid Mech., Vol.24 (1966), pp.41-63.

(26) Tomiyama, A. and Hirano, M., An Improvement of the Computational Efficiency of the SOLA Method, JSME Int. J., Ser. B, Vol.37, No.4 (1994), pp.821-826.

(27) Amsden, A.A. and Harlow, F.H., The SMAC method: A Numerical Technique for Calculating Incompressible Fluid Flows, LA-4370, Los Alamos Scientific Laboratory Report, (1970).

(28) JSME, Thermophysical Properties of Fluids, The Japan Society of Mechanical Engineers, Tokyo, (1997). 